\title{
Wideband polarization-independent anomalous reflection metasurface with multiple resonance modes
}

\author{
Guoxiang Dong* ${ }^{* \dagger}$, Song Xia* ${ }^{* \dagger}$, , Yongyong Zhuang*,†, Hongyu Shi*, Zhan Zhang*, \\ Yuchen $\mathrm{He}^{*, \dagger}$, Anxue Zhang ${ }^{*, \dagger}$, Xiaoyong Wei*, ${ }^{*}$, and Zhuo $\mathrm{Xu}^{*, \dagger}$ \\ *Electronic Materials Research Laboratory, Key Laboratory of the Ministry \\ of Education \& International Center for Dielectric Research \\ Xi'an Jiaotong University \\ Xi'an, Shaanxi 710049, P. R. China \\ ${ }^{\dagger}$ School of Electronic and Information Engineering \\ Xi'an Jiaotong University \\ Xi'an, Shaanxi 710049, P. R. China \\ *Zhonghang Electronic Measuring Instruments Co., Ltd \\ Xi'an, Shaanxi 710049, P. R. China

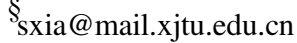

Received 21 February 2017; Revised 16 March 2017; Accepted 31 March 2017; Published 26 April 2017

\begin{abstract}
An ultra-thin metasurface is proposed to realize wideband polarization-independent anomalous reflection. The sub-wavelength resonator can produce different resonance modes, which are the result of the combined effect of dielectric and the metallic structure. The gradient metasurface is done by six discrete orientation of the local sub-wavelength resonator which provides a phase gradient. The simulation and measured results show that $9 \mathrm{GHz}$ bandwidth of the anomalous reflection is achieved.
\end{abstract}

Keywords: Metasurface; anomalous reflection; polarization.

\section{Introduction}

Metasurface is the planar version of metamaterial, ${ }^{1-3}$ which can provide abrupt phase change to give more freedom of controlling the propagation of electromagnetic wave. Subwavelength resonators are usually used to provide abrupt phase change. Many kinds of arrangements of resonators can be designed to achieve many novel physical efforts ${ }^{4}$ or components, such as polarization rotation, ultra-thin cloak, planar lens, anomalous reflection/refraction and surface wave coupling. When the arrays of the resonators can provide a constant gradient of the phase discontinuity, such a metasurface is called phase gradient metasurface. ${ }^{5-7}$

Surface wave coupling and anomalous reflection are fascinating physical efforts, which can be realized simultaneously by the gradient metasurface. The generalized Snell law can be used to describe that, which is firstly proposed by $\mathrm{Na}$ fang Yu et al. Surface wave coupling is not easy to achieve by conventional components. Bulky grating or dielectric prisms which require oblique incident with a special angle to meet the large wave vector required by surface wave are usually used, but the efficiency is quite low. However, surface wave coupling and anomalous reflection can be achieved by the PGMs with normal incidence and have high efficiency. The PGMs can provide an additional transverse wave vector, which can either be greater or smaller than that of EM wave. So the incident wave can be coupled into surface wave or reflected anomalously, respectively. The PGMs composed of $V$-shaped nanoantennas are firstly observed anomalous refraction, the PGMs composed of $H$-shaped resonators are firstly observed surface wave coupling. In the subsequent work, many kinds of PGMs have been proposed, including $u$-shaped, $c$-shaped and split-ring resonators. However, these PGMs can only work under a specific polarization. In contrast, when the abrupt phase change is Pancharatnan-Berry phase, the PGM is not limited by the polarization state. Wideband anomalous reflection can be realized by these PGMs. A transmission PGM was proposed to realize anomalous refraction and surface wave coupling at specific frequency. In conclusion, these PGMs also suffer some drawbacks, for example polarization angle-dependent, only realizing anomalous reflection and surface wave coupling, respectively, working at only single frequency point. So, a PGM which can realize wideband polarization-independent surface wave coupling and anomalous reflection simultaneously is still highly desirable.

In this paper, we proposed a wideband polarizationindependent anomalous reflection metasurface, which is a phase gradient metasurface. Under normal linear incidence, wideband anomalous reflection is realized, and the reflection

This is an Open Access article published by World Scientific Publishing Company. It is distributed under the terms of the Creative Commons Attribution 4.0 (CC-BY) License. Further distribution of this work is permitted, provided the original work is properly cited. 
wave is symmetrical. Both the simulated and experimental results show that $9 \mathrm{GHz}$ bandwidth of the anomalous reflection can be achieved simultaneously, and the efficiency is higher.

\section{The Metasurface Design and Simulation}

\subsection{Metasurface design}

Figure 1 shows unit cell structure of the designed ultra-thin metasurface. The geometrical parameters of the unit cell are given by $p=3 \mathrm{~mm}, r_{1}=1.2 \mathrm{~mm}, r_{2}=0.8 \mathrm{~mm}, w=0.2 \mathrm{~mm}$. The thickness and the dielectric constant of the substrate is $1.5 \mathrm{~mm}$ and 4.4, respectively. The other side of the substrate is covered fully with metallic sheet.

Figure 2 gives the simulated same handedness (s11) reflectivity and opposite handedness (s21) reflectivity of metasurface reflector under normally incident LCP and RCP waves. It can be seen from Fig. 2 that over a wide frequency

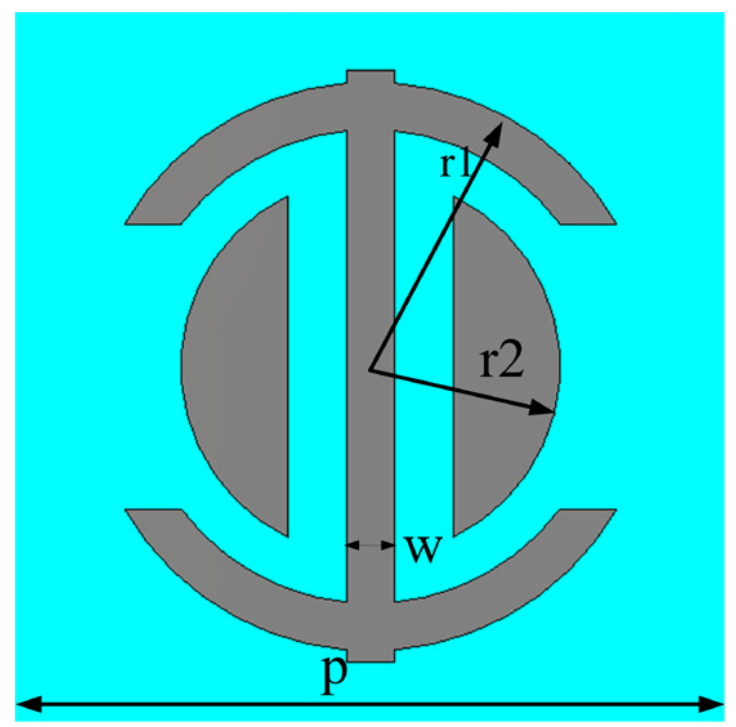

Fig. 1. The unit cell.

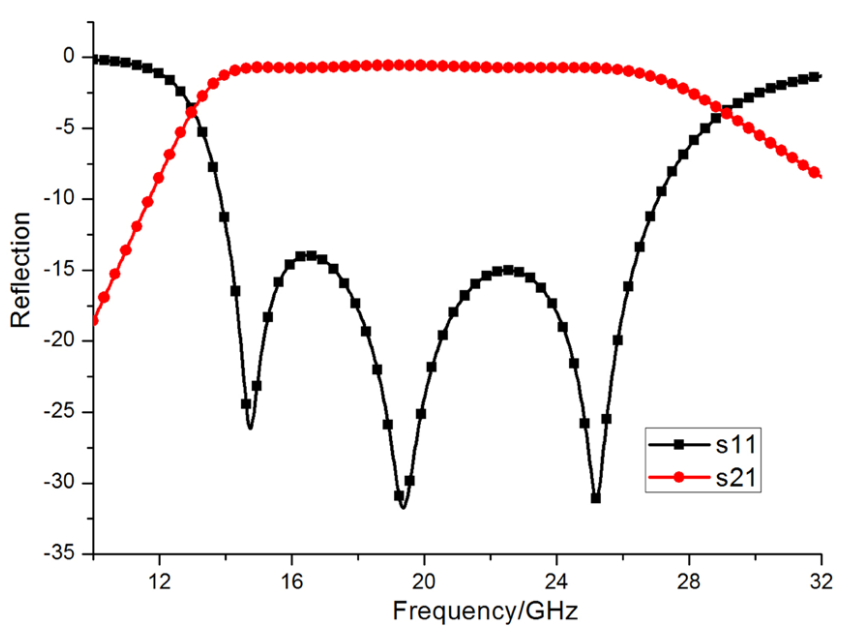

Fig. 2. The simulated refectivity for $\mathrm{CP}$ incidence. range, the opposite handedness reflectivity is very high. The high-efficiency opposite handness reflectivity mainly results from the different resonance of the unit resonator. From the simulated reflectivity under $\mathrm{CP}$ wave incidence, we find that there are three resonance frequencies located at 15.049, 19.255 and $24.97 \mathrm{GHz}$. The $E_{z}$ distribution of three resonance frequencies are shown in Fig. 3. We can find that these resonance frequencies have different resonance modes. These resonances are the result of the combined effect of dielectric and the metallic structure. The wide operation bandwidth is produced due to the superimposition of the three resonance modes.

The metasurface consists of an array of the unit cell with the same geometry but spatially varying orientations. When a beam of circularly polarized light is incident onto the metasurface, the reflection wave is partially converted into the opposite handedness of CP light with a phase change determined solo by the orientation of the unit cell, when the incident wave is

$$
\mathbf{E}_{i}=(\mathbf{x}+j \mathbf{y}) e^{-j k z} e^{-j w t}
$$

when the unit cell is rotated by $\varphi$, the reflection wave can be expressed:

$$
\begin{aligned}
\mathbf{E}_{r}= & 1 / 2\left[\left(R_{x}-R_{y}\right)(\mathbf{x}-j \mathbf{y}) e^{j 2 \varphi}+\left(R_{x}+R_{y}\right)(\mathbf{x}+j \mathbf{y})\right] \\
& \times e^{j k z} e^{-j w t} .
\end{aligned}
$$

Therefore, we can modulate the wave front for the opposite handedness light by a phase discontinuity $2 \varphi$. The phase discontinuity is twice that of the rotation angle of the unit cell. Obviously, the phase discontinuity of the opposite handedness light is $-2 \varphi$ for the left incident wave. This is a simple linear relationship between the phase discontinuity and the rotation angle of the unit cell. The proposed metasurface is shown in Fig. 4, which comprised six unit cell with different orientations. The reflectivity of the unit cell with different orientations for circularly polarized incidence is shown in Fig. 5. The results show that the reflection is higher than 0.9 for wideband. Furthermore, the curves of the reflectivity of the different orientation unit cells are completely coincident because the metasurface is an array of the unit cell with different orientations. Figure 6 show the simulation results of the reflection phase of the six unit cells under normal circularly polarized wave, which indicate that the phase gradient is opposite for left- and right-handed circularly polarized wave.

\subsection{Simulation results}

The phase gradient metasurface is composed of six different orientations of the resonators shown in Fig. 1, the relative rotation angle is $30^{\circ}$ between the neighboring resonators, as shown in Fig. 4. So, the gradient of phase variation is $\xi= \pm \Delta \varphi / P$, where the sign is decided by the helicity of the incident circularly polarized wave. The wave vector along PGM surface is $k=\xi+k_{0} \sin \theta$, where $k_{0}$ is the wave vector 


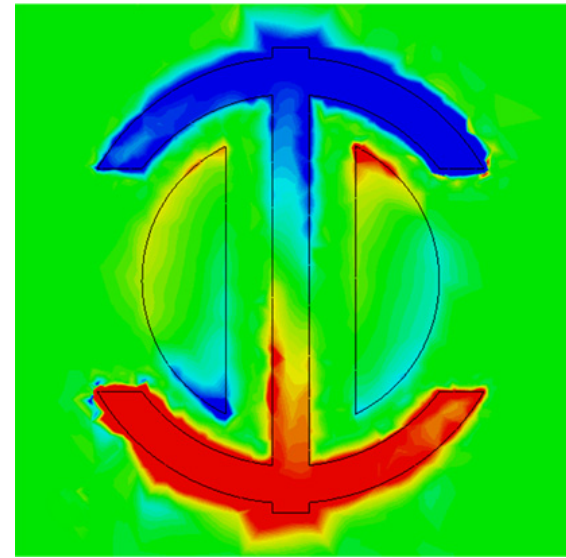

(a)

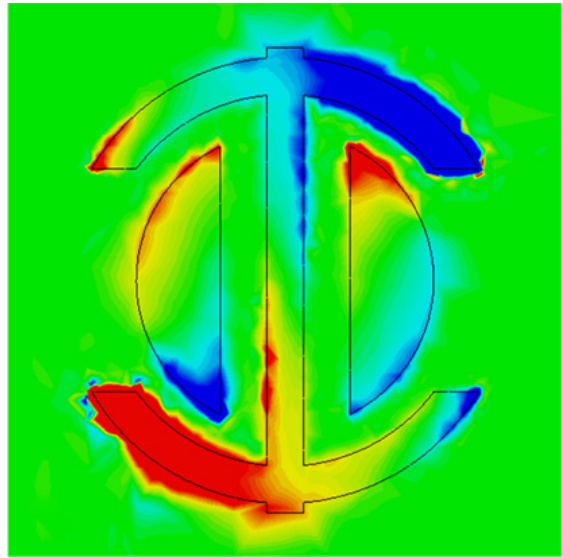

(b)

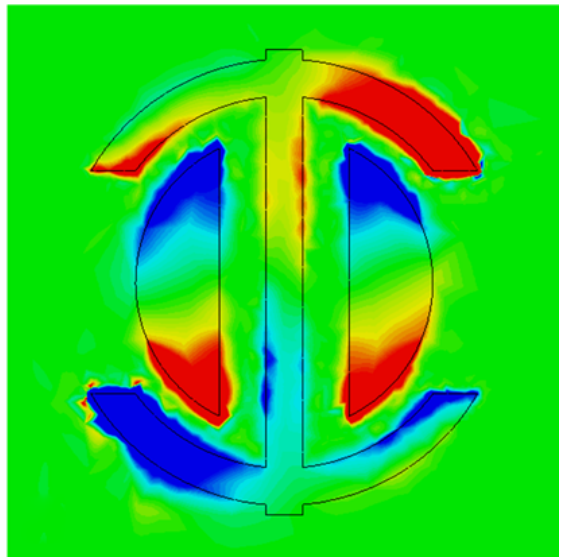

(c)

Fig. 3. The $E_{z}$ distrution at (a) $15.049 \mathrm{GHz}$, (b) $19.255 \mathrm{GHz}$ and (c) $24.97 \mathrm{GHz}$.

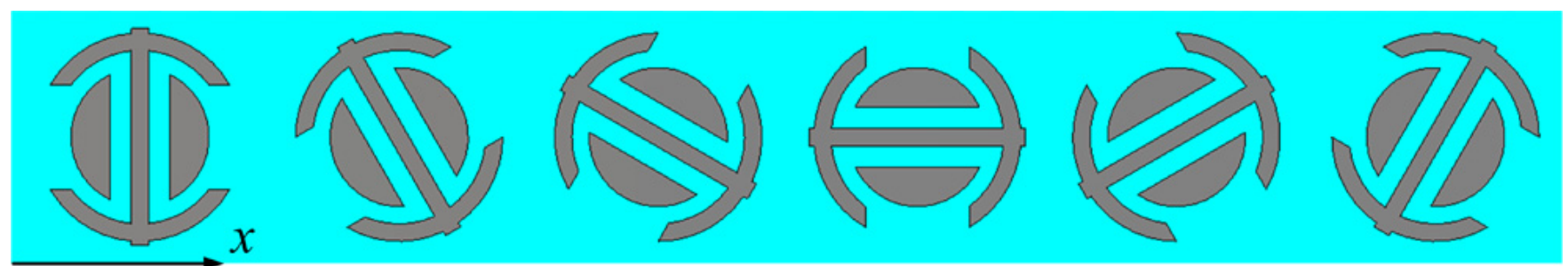

Fig. 4. The proposed metasurface.

of the incident wave. $\theta$ is the angle of incident wave. The wave vector provided by the metasurface have opposite direction for different helicity circularly polarized wave.

Under normal incidence, the wave vector along the PGM is $k=\xi$, which is completely provided by the PGM. When $|\xi|>k_{0}$, the incident wave is coupled into the surface wave, which propagates along the surface of the PGM and can not be detected above the surface of the PGM. This physical effort is described by the generalized Snell law. When

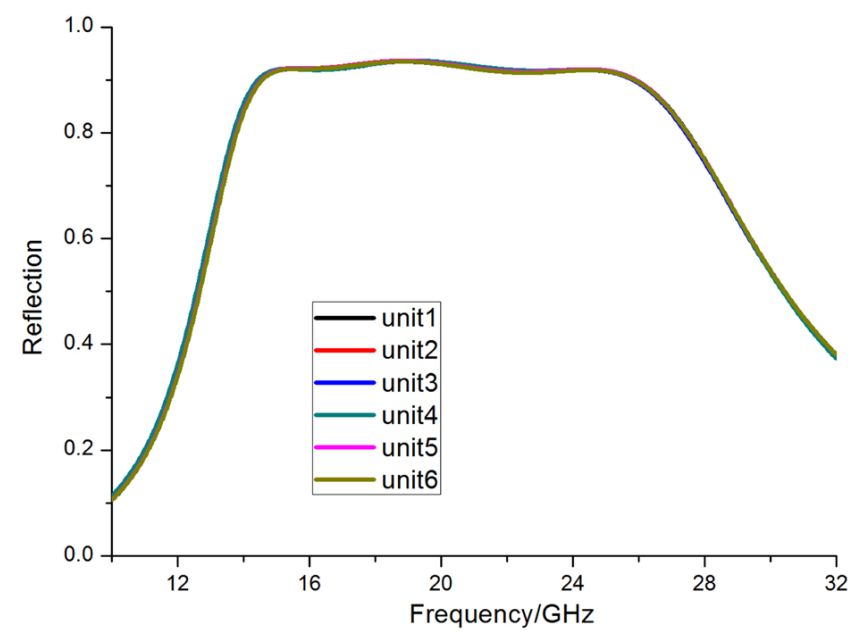

Fig. 5. Reflection of the unit cell. $|\xi|<k_{0}$, the incident wave is reflected anomalously and the reflection angle is $\theta_{r}=\arcsin \left(\xi / k_{0}\right)$.

From $17 \mathrm{GHz}$ to $26 \mathrm{GHz}$, the condition $|\xi|<k_{0}$ is satisfied. Thus, the anomalous reflection is achieved from $17 \mathrm{GHz}$ to $26 \mathrm{GHz}$. In order to verify the wideband anomalous reflection, the anomalous reflection at $22 \mathrm{GHz}$ is observed. In the simulation, the incident wave is linearly polarized wave, which can decompose into left- and right-handed circularly polarized wave. The proposed metasurface provides opposite

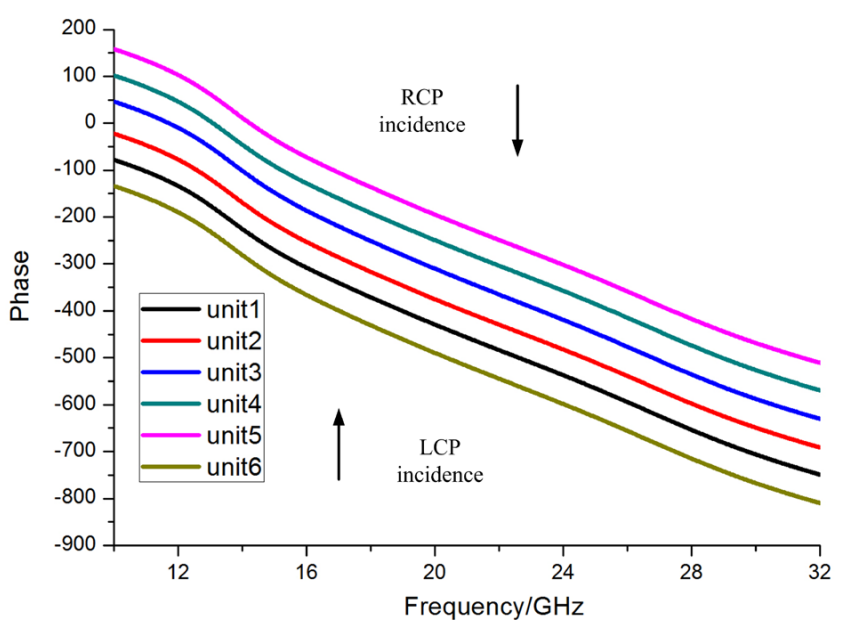

Fig. 6. The phase distribution of the unit cell. 


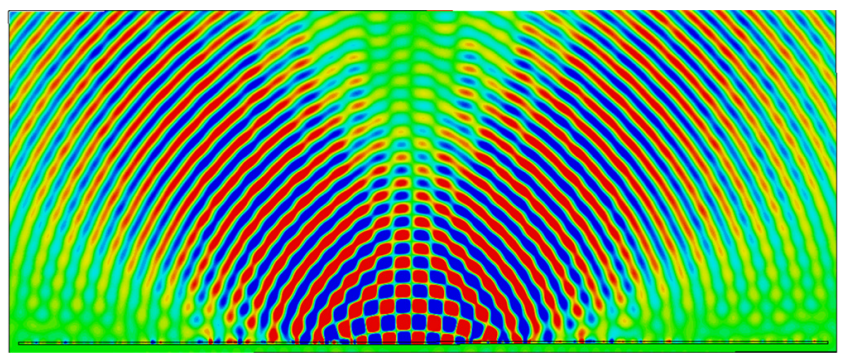

Fig. 7. The anomalous reflection at $22 \mathrm{GHz}$.

phase gradient, so the reflection wave shown in Fig. 7 is anomalous and symmetrical for left- and right-handed circularly polarized incident wave.

\section{Measurement Result}

A $410 \mathrm{~mm} * 410 \mathrm{~mm}$ sample of the metasurface shown in Fig. 8 was fabricated to further verify the design through measurements. The reflection measurement setup is shown in Fig. 9. Two horn antennas are used: one to radiate an EM wave onto the sample metasurface, and another to receive the reflected waves from the sample. The two horn antennas are kept at the same height of the centers of the sample to ensure that the wave can be reflected and received by the sample.

The measured specular reflectivity for the $x$-polarized waves under normal incidence is given in Fig. 9. It can be found that the specular reflectivity is significantly decreased. The reflectivity is below $-15 \mathrm{~dB}$ over a wide frequency range from $17 \mathrm{GHz}$ to $26 \mathrm{GHz}$, which is caused by the wideband anomalous reflection.

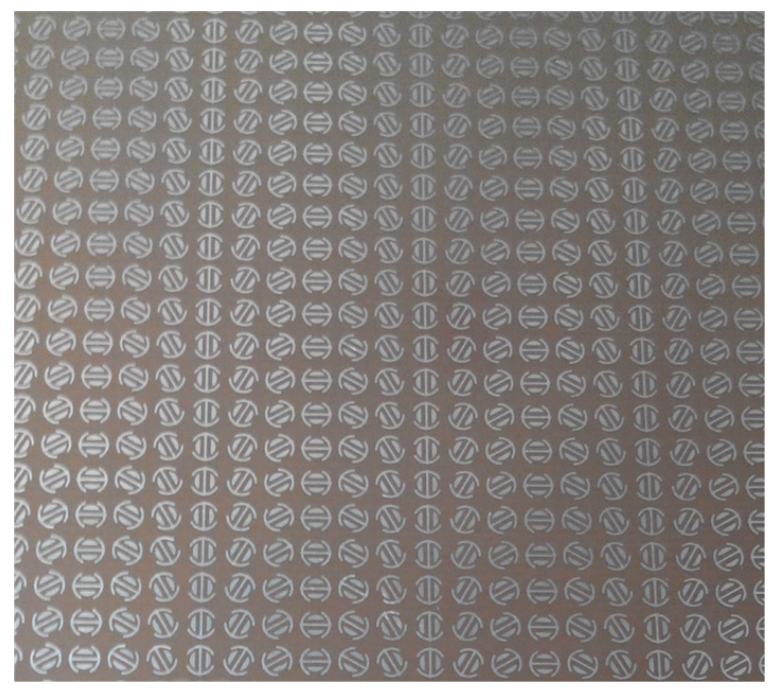

Fig. 8. A fabricated sample of the metasurface.

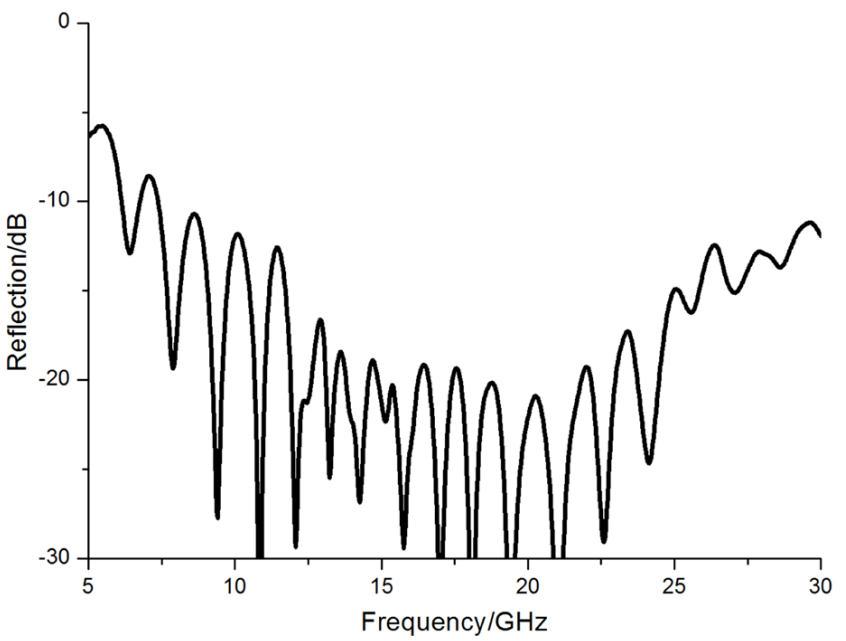

Fig. 9. The measured reflectivity of the phase gradient metasurface under LP wave illumination.

\section{Conclusion}

In summary, the wideband polarization-independent anomalous reflection is realized using PGMs for CP waves. To obtain high-efficiency anomalous reflection, we designed the proposed metasurface, which provides opposite phase gradients with the same amplitude LCP and RCP waves. Under LP incident wave, the reflected waves are divided into two $\mathrm{CP}$ waves, and the anomalous reflection is symmetrical. the anomalous reflection is polarization-independent of incident LP waves.

\section{References}

${ }^{1}$ F. Aieta, P. Genevet, N. F. Yu, M. A. Kats, Z. Gaburro and F. Capasso, Out-of-plane reflection and refraction of light by anisotropic optical antenna metasurfaces with phase discontinuities, Nano Lett. 12, 1702 (2012).

${ }^{2}$ C. Pfeiffer and A. Grbic, Cascaded metasurfaces for complete phase and polarization control, Appl. Phys. Lett. 102, 231116 (2013).

${ }^{3}$ M. Farmahini-Farahani and H. Mosallaei, Birefringent reflectarray metasurface for beam engineering in infrared, Opt. Lett. 38, 462 (2013).

${ }^{4}$ H. F. Ma, G. Z. Wang, G. S. Kong and T. J. Cui, Broadband circular and linear polarization conversions realized by thin birefringent reflective metasurfaces, Opt. Mater. Exp 4, 1717 (2014).

${ }^{5}$ Y. Liu, X. Ling, X. Yi, X. Zhou, H. Luo and S. Wen, "Realization of polarization evolution on higher-order Poincare sphere with metasurface, Appl. Phys. Lett. 104, 191110 (2014).

${ }^{6}$ J. Y. Chin, M. Lu and T. J. Cui, Metamaterial polarizers by electricfield-coupled resonators, Appl. Phys. Lett. 93, 257903 (2008).

${ }^{7} \mathrm{P}$. Mingbo et al., Anisotropic meta-mirror for achromatic electromagnetic polarization manipulation, Appl. Phys. Lett. 102, 131906 (2013). 